\title{
Impacto do controle do mofo-branco com fluazinam na produtividade da soja no Sul do Paraná: metanálise
}

\author{
Fernando Luiz Buss Tupich, Lucas Henrique Fantin, André Luis da Silva, Marcelo Giovanetti Canteri
}

Universidade Estadual de Londrina, Departamento de Agronomia, Caixa Postal 6001, CEP 86051-970, Londrina,PR

Autor para correspondência: Lucas Henrique Fantin (fantinagro@gmail.com)

Data de chegada: 13/09/2016. Aceito para publicação em: 16/02/2017.

\section{RESUMO}

$10.1590 / 0100-5405 / 168479$

Tupich, F.L.B.; Fantin, L.H.; Silva, A.L.; Canteri, M.G. Impacto do controle do mofo-branco com fluazinam na produtividade da soja no Sul do Paraná: metanálise. Summa Phytopathologica, v.43, n.2, p.145-150, 2017.

O controle químico de Sclerotinia sclerotiorum pode prevenir infecção, e reduzir a incidência e severidade da doença. Analisar resultados de ensaios individuais nem sempre fornecem conclusões confiáveis. Com isso, técnicas estatísticas como a metanálise, pouco utilizada na fitopatologia, podem ser úteis. Basicamente a técnica sumariza quantitativamente resultados de diversos estudos os ponderando de acordo com sua variabilidade. Assim, foram analisados trabalhos publicados e relatórios técnicos fornecidos por entidades de pesquisa, contendo o tratamento químico com o ingrediente ativo fluazinam usado no controle de S. sclerotiorum e seu efeito na produtividade da soja. Foram analisados 18 experimentos, totalizando 50 entradas de dados. A variável utilizada para o modelo de efeitos aleatórios foi obtida pela diferença entre o tratamento com o fungicida e a testemunha (sem aplicação). A estimativa metanalítica indicou acréscimo de 413,9 kg.ha ${ }^{-1}$ com amplitude de 344,6 a $483,1 \mathrm{~kg} \cdot \mathrm{ha}^{-1}$. A probabilidade de haver qualquer acréscimo de produtividade foi de $89,1 \%$, sendo que para 120,300 , 600,900 e $1.200 \mathrm{Kg}$.ha-1 as chances foram 82,8; 69,6; 41,7; 17,6 e 4,9\%, respectivamente. Considerando-se $\mathrm{R} \$ 50,00$ o preço da saca $60 \mathrm{~kg}$ e $\mathrm{R} \$ 170,00$. ha $^{-1}$ o custo do fungicida, estimou-se em $77,1 \%$ a probabilidade de retorno econômico. Concluiu-se que a aplicação de fluazinam contribuiu para o aumento de produtividade de soja atacada por S.scleotiorum.

Palavras-chave: Sclerotinia sclerotiorum, modelo de efeitos aleatórios, Revisão sistemática.

\section{ABSTRACT}

Tupich, F.L.B.; Fantin, L.H.; Silva, A.L.; Canteri, M.G. Impact of white mold control by fluazinam on soybean yield in the south of Paraná: Metaanalysis. Summa Phytopathologica, v.43, n.2, p.145-150, 2017.

Chemical control of Sclerotinia sclerotiorum can prevent infection and reduce the disease incidence and severity. Assessment of individual assay results may not provide reliable conclusions. Statistical techniques like metaanalysis, rarely adopted in phytopathology, may be useful. Basically, the technique quantitatively summarizes the results of several studies, weighting them according to their variability. Thus, published studies and technical reports provided by research entities were analyzed; they contained chemical treatment with the active ingredient fluazinam used in S. sclerotiorum control and its effect on soybean yield. Eighteen experiments were analyzed, totaling

Keywords: Sclerotinia sclerotiorum, random effects model, systematic review.
50 data entries. The variable used for the random effects model was obtained based on the difference between the fungicide treatment and the control (without application). Meta-analytic estimate indicated an increase of $413.9 \mathrm{~kg} \cdot \mathrm{ha}^{-1}$ and range from 344.6 to $483.1 \mathrm{~kg} \cdot \mathrm{ha}^{-1}$. Probability of any increase in yield was $89.1 \%$, and for $120,300,600,900$ and 1,200 Kg.ha ${ }^{-1}$ chances were 82.8, 69.6, $41.7,17.6$ and $4.9 \%$, respectively. Considering R $\$ 50.00$ is the cost of a $60 \mathrm{~kg}$ bag and $\mathrm{R} \$ 170.00 . \mathrm{ha}^{-1}$ is the fungicide cost, the probability of financial return was estimated at $77.1 \%$. Fluazinam application contributed to increase the yield of soybean attacked by $S$. sclerotiorum.
O estado do Paraná é o segundo maior produtor nacional de soja, com cerca de 17 milhões de toneladas, e produtividade média de 3141 kg.ha- ${ }^{-1}(7)$. Entre as regiões produtoras de soja no estado, a região centro-sul é responsável por quatro milhões de toneladas (23). A presença de mofo branco nas lavouras de soja da região tem contribuído para a ocorrência de reduções significativas na produção da soja.

O fungo Sclerotinia sclerotiorum Lib. de Bary, agente causal do mofo branco da soja, é um patógeno necrotrófico e cosmopolita, podendo infectar mais de 400 hospedeiros (4). As condições ideias para o desenvolvimento do patógeno são temperaturas moderadas, entre $18 \mathrm{e}$ $23^{\circ} \mathrm{C}$, associadas à alta umidade relativa do ar, ocorrentes em zonas de clima temperado, regiões subtropicais ou tropicais de altitude elevada (25), características da região centro-sul do Paraná.

A sobrevivência do inóculo no solo ocorre na forma de escleródios do fungo, assegurandonovas infecções em safras posteriores, além da disseminação para novas áreas via infestação de sementes e em maquinários $(2,3,10,12)$. Segundo Meyer et al. (14) em 2013, cerca de $19 \%$ das áreas de produção de soja no estado do Paraná estavam infestados pelo patógeno.

O controle do mofo branco da soja apesar de estar principalmente relacionada a métodos culturais como rotação de culturas, escolha da época de semeadura, espaçamento e densidade de plantas, pode também ser realizado através do controle químico com fungicidas no tratamento de sementes e aplicação nos órgãos aéreos, visando a redução da intensidade da doença na parte área. Dentre os fungicidas utilizados nos ensaios em rede de pesquisa visando o controle do mofo-branco, liderada pela EMBRAPA-Soja, o ingrediente ativo fluazinam destacouse por sua eficiência no controle do fungo $(14,15)$.

Contudo, resultados obtidos em estudos com o controle químico de $S$. sclerotiorum nem sempre apresentam efeito significativo 
( $p>0,05)$, reduzindo a confiabilidade nos resultados. Salam et al. (22) estudando o efeito do controle químico de doenças em trigo, citam que experimentos individuais demonstram o efeito do controle nas condições especificas do ensaio e que a interpretação individual não fornecem uma resposta global de produtividade em reposta confiável do controle com fungicidas. Desta forma a análise conjunta de ensaios é uma ferramenta valiosa necessária para fornecer estimativas que sob análise de ensaios individuais não seriam fornecidas (1).

Dentre as técnicas de sumarização de dados Scherm et al. (24) citam o data mining (extração e identificação de padrões entre dados) e a metanálise (resumo quantitativo de resultados). Essa técnica consiste na revisão sistemática de estudos individuais, onde a variabilidade de cada ensaio fornece seu nível de contribuição na estimativa global. Basicamente, os dados são ponderados de acordo com modelos, podendo ser modelos de efeitos fixos e de efeitos aleatórios.

O modelo de efeitos fixos considera que todos os ensaios sofrem a mesma influência e que a diferença entre os resultados observados é oriunda do erro amostral. Já o modelo de efeitos aleatórios entende que as diferenças entre os resultados apresentados sofrem influências diferentes devido às características especificas de cada experimento, por exemplo o local. Esta variação pode ser explorada pela heterogeneidade, analisada através do modelo de efeitos mistos, sendo adotadas variáveis moderadoras, ou subgrupos que venham a influenciar as estimativas dos efeitos $(5,8,11,16,21)$.

Assim, o presente trabalho teve por objetivo quantificar através de modelos metanalíticos, a resposta em produtividade da aplicação foliar do ingrediente ativo fluazinam no controle de S. sclerotiorum e estimar a probabilidade de acréscimo de produtividade em resposta ao controle obtido.

\section{MATERIAL E MÉTODOS}

\section{Revisão sistemática e seleção de dados}

A coleta de dados foi realizada através do levantamento de trabalhos utilizando o controle químico de mofo branco na cultura da soja com aplicação foliar do ingrediente ativo fluazinam e a resposta em produtividade. As bases utilizadas foram Scopus e CAB utilizando a combinação das palavras chemical control, Fluazinam e white mold. Os critérios para inclusão dos estudos foram: 1) Experimentos apresentando o controle químico de mofo branco da soja através de aplicação foliar e resposta em produtividade; 2) Trabalhos conduzidos na região central-sul do Paraná 3) Estudos apresentando concentração de ingrediente ativo aplicado, época e número de aplicações; 4) Trabalhos que apresentavam informado medidas de variabilidade e dispersão de dados como coeficiente de variação (CV) e número de repetições. Devido ao baixo número de trabalhos publicados ou que não atendiam os parâmetros estabelecidos, como o caso do estudo de Wutzki et al. (29) conduzido na região mas sem apresentar medidas de variabilidade, foram incluídos na análise parte dos dados utilizados na dissertação de Tupich (27).

\section{Pré-analise}

A normalidade dos resultados dos experimentos foram testados a partir do teste de Shapiro-Wilk e elaborados os gráficos de distribuição de frequência e histograma com intuito de avaliar visualmente o comportamento dos dados.

\section{Medidas descritivas}

A variável resposta utilizada, denominada medida de efeito $(D)$, foi obtida a partir da diferença entre a produtividade do tratamento com fungicida e a testemunha sem aplicação. Assim como utilizada por Paul et al. (19) estudando o efeito da controle químico de doenças em milho.

\section{$\mathbf{D}=($ Prod. Trat - Prod. Test $)$}

Onde, Prod trat é a média de produtividade do tratamento com aplicação foliar do ingrediente ativo fluazinam e Prod test é a média de produtividade da testemunha sem aplicação.

A variabilidade dos dados inseridos na metanálise foi estimada pelo erro padrão. Calculou-se primeiramente o desvio padrão, a partir o coeficiente de variação $(\mathrm{CV})$ apresentado em cada estudo, utilizando-se como base, as médias de produtividade dos tratamentos com fungicida e testemunha:

\section{$\sigma=(($ Prod. Trat + Prod. Test $) / 2 * C . V) / 100$}

Onde, $\sigma$ é o desvio padrão e $\boldsymbol{C}$. $\boldsymbol{V}$ é o coeficiente de variação.

Para cada entrada de dado utilizada, estimou-se ainda seu respectivo erro padrão

\section{Erro padrão $=\sigma / \sqrt{ } n$}

n é o número de repetições do experimento.

A partir do erro padrão e medida de efeito, efetuou-se a metanálise utilizando o modelo que melhor explica a variação de efeitos dos dados. Os modelos foram desenvolvidos no programa $\mathrm{R}$ (20), pacote de dados Metafor (28).

\section{Modelo de efeitos aleatórios}

Considerando que o conjunto de estudos tem distribuição normal com o efeito médio $(\mu)$ e variância do efeito $\left(\tau^{2}\right)$ representado pela notação $\theta_{i} \sim N\left(\mu, \tau^{2}\right)$ (27), o modelo de efeitos aleatórios utiliza como fontes causadoras de efeito a variação dentro do estudo (within-study) e entre os experimentos (between-study), Podendo ser escrito como:

$$
\mathrm{Y}_{j}=\mu+\xi_{j}+\varepsilon_{j}
$$

Onde $\mathrm{Y}_{j}$ representa o efeito observado no estudo $\mathrm{j}, \mu$ é o efeito comum a todos os estudos, ou a medida sumarizada ou medida metanalítica, $\varepsilon \mathrm{j}$ corresponde ao erro aleatório do estudo j e $\xi_{j}$ é a variação nos efeitos verdadeiros. Para obter a estimativa global e minimizar a variância, calculou-se a média ponderada, em que o peso atribuído a cada entrada foi dado pelo inverso de sua variância, a partir da variância dentro do estudo e entre estudos.

\section{Heterogeneidade}

Para se determinar o impacto da heterogeneidade da estimativa global, foram calculados os índices $\mathrm{H}^{2}$ e $\mathrm{I}^{2}$ propostos por Higgins \& Thompson (9). O índice $\mathrm{I}^{2}$ fornece a porcentagem total de variância que é devido a heterogeneidade entre estudos. $\mathrm{H}^{2}$ faz uma relação entre o tamanho do intervalo de confiança e a estimativa da medida de efeito. $\mathrm{H}^{2}$ maior que dois indica que a variação entre estudos possui influencia substancial no efeito global.

\section{Moderadoras de efeito}

De acordo com os diferentes níveis de incidência e severidade contidos no tratamento testemunha dos estudos inseridos na metanálise, foram estabelecidos subgrupos que permitissem aumentar o conhecimento sobre o assunto. A segmentação em subgrupos foi realizada para verificar a influência dos diferentes níveis de incidência e severidade sobre a resposta em produtividade.

Os critérios para divisão de entradas foram, 1) Estudos com a aplicação de fluazinam no controle de mofo branco da soja, 
apresentando valores de produtividade, medidas de dispersão e severidade; 2) Experimentos com a aplicação de fluazinam no controle de mofo branco da soja, apresentando valores de produtividade, medidas de dispersão e incidência.

\section{Probabilidade de incremento em Produtividade}

A partir das estimativas obtidas pelo modelo de efeitos aleatórios foi determinado a probabilidade de ganho seguindo a metodologia proposta por Paul et al. (17) e Madden e Paul (11). Onde a estimativa da probabilidade é dada por:

$$
p_{\vartheta}=\operatorname{Pr}\left(\zeta_{\text {new }}>\vartheta\right)=1-\phi\left(\frac{\vartheta-\zeta}{\sigma}\right)
$$

Sendo que, $\zeta$ new indica o efeito do tratamento a ser estimado, $\vartheta$ é a resposta da produtividade a ser estimada, $\sigma$ variância entre os estudos, $\zeta$ estimativa metanalítica e $\phi$ é função da Distribuição Normal. Foram estipulados probabilidade de retorno positivo da produtividade para os níveis 120, 300, 600, 900 e $1.200 \mathrm{Kg}$ ha $^{-1}$ de acréscimo, para tratamentos com a aplicação do ingrediente ativo fluazinam em soja sob influência de mofo branco. Os valores de probabilidade foram multiplicados por 100 e expressos em porcentagem.

\section{Retorno financeiro}

Ainda, seguindo-se a mesma metodologia de probabilidade de incremento de produtividade, calculou-se o beneficio econômico referente a resposta em produtividade, assim como utilizado por Salam et al (22). A base para o cálculo seguiu o preço da saca de soja e o custo. ha $^{-1}$ da aplicação. Com base no custo total de aplicação em sacas. haestimou-se a probabilidade de retorno financeiro.

\section{RESULTADOS E DISCUSSÃO}

\section{Levantamento de dados}

A partir dos critérios de seleção de trabalhos, foram levantados 18 estudos, totalizando 50 resultados (entradas). Do total de entradas avaliadas, oito apresentavam apenas uma aplicação de fluazinam próximo ao início do período reprodutivo, 38 apresentavam duas aplicações (V9 e R1 a R3) e três ensaios apresentando três aplicações (R1, 10 e 20 dias após R1). Os ensaios utilizados foram realizados nos municípios de Castro, Ponta Grosa, Arapoti, Mauá da Serra, Palmeira e Marilândia do Sul (Figura 1).

\section{Pré-análise}

Os dados levantados foram agrupados e a partir da medida de efeito (D) e testada a normalidade pelo teste de Shapiro-wilk e plotado gráfico de distribuição de frequência com barras de erro padrão (Figura 1). O gráfico apresenta visualmente o comportamento do efeito da aplicação de fluazinam na produtividade de soja.

Do total, 45 entradas apresentaram acréscimo $\left(\mathrm{D}>0 \mathrm{Kg}_{\mathrm{h}} \mathrm{ha}^{-1}\right)$ e cinco entradas indicaram decréscimo de produtividade $\left(\mathrm{D}<0 \mathrm{Kg}^{-h^{-1}}{ }^{-1}\right.$. Contudo, das 39 entradas que apresentaram acréscimo de produtividade $(\mathrm{D}>0), 14$ não apresentaram diferença significativa $(\mathrm{p}<0.05)$ em seus estudos originais.

\section{Modelo de efeitos aleatórios}

Segundo Borenstein (5) o modelo de efeitos aleatórios considera que o efeito atribuído a cada ensaio não é mesmo para todos. Assim, a estimativa final de efeito é dada pelo efeito médio, de forma que o resultado final é dado em estimativa, conhecida como estimativa metanalítica. Os resultados apresentaram resposta significativa $(\mathrm{p}<0.001)$ de acréscimo de produtividade, com estimativa de 413,9 kg.ha ${ }^{-1}$ e amplitude variando de $344,6 \mathrm{~kg} \cdot \mathrm{ha}^{-1} \mathrm{a} 483,1 \mathrm{~kg} \cdot \mathrm{ha}^{-1}$, ao ser utilizado fluazinam no controle de mofo branco da soja (Tabela 1).

$\mathrm{O}$ incremento de produtividade com aplicação de fluazinam confirmam os estudos realizados por Meyer et al. (13) que observaram acréscimo de produtividade significativa $(\mathrm{p}<0,05)$. Calvo et al. (6) trabalhando com métodos metanalíticos também apresentaram acréscimo de produtividade com o controle químico. No estudo os autores levaram em consideração diferentes ingredientes ativos no controle de S.sclerotiorum. Já Wutzki et al (29) em ensaios conduzidos na região central do Paraná não verificaram diferença estatística significativa para produtividade, contudo citam que o controle químico reduziu significativamente a incidência e produção de escleródios nos locais com maior incidência ( $31 \%$ e $29,8 \%)$. Visto que um escleródio tem a capacidade de produção de até dois milhões de ascósporos no

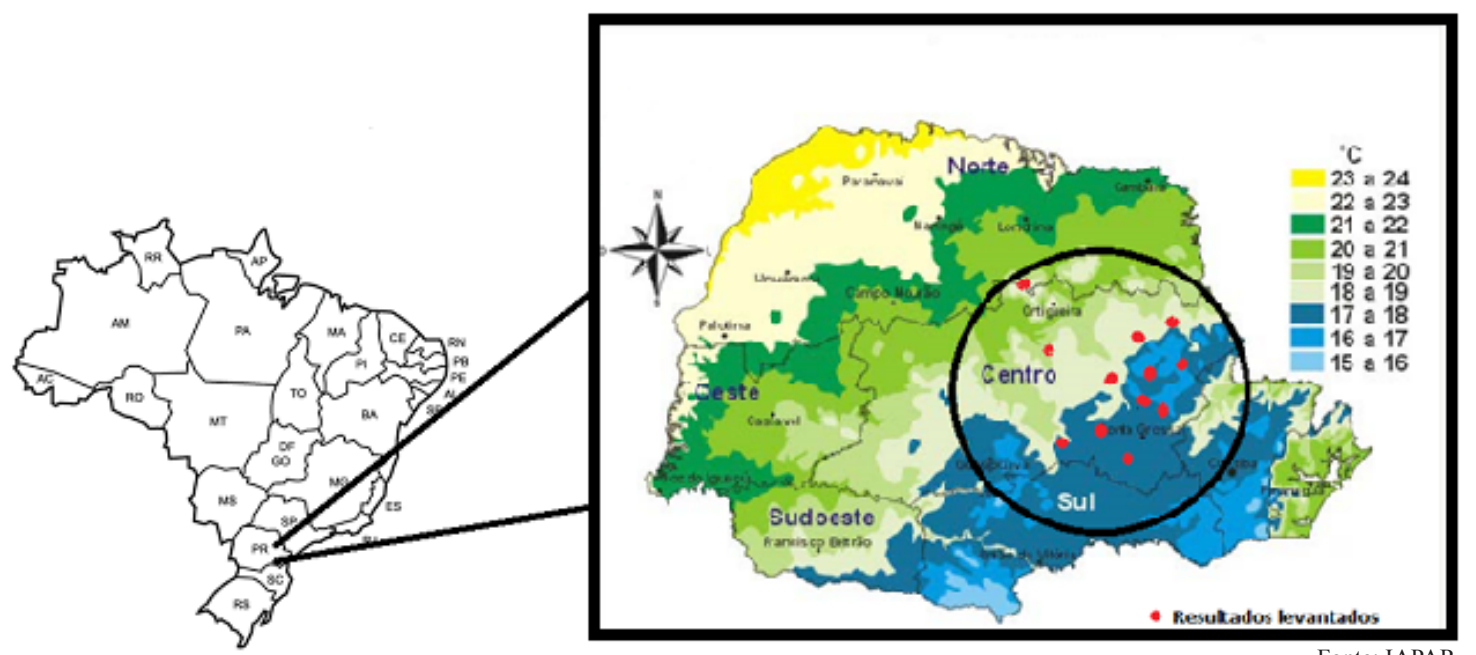

Figura 1. Distribuição dos pontos experimentais em divisão por faixas de temperaturas anuais médias dos ensaios de campo conduzidos com e sem tratamento do fungicida fluazinam para controle de Sclerotinia sclerotiorum. 


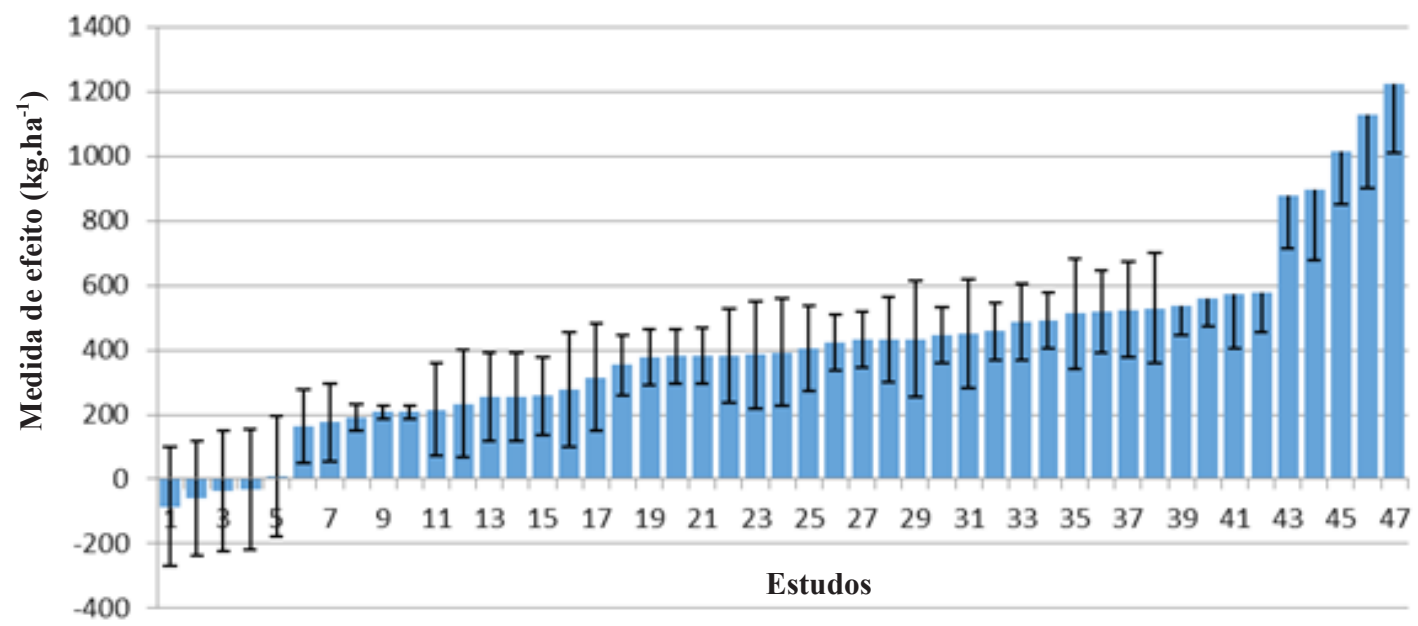

Figura 2. Distribuição do efeito da aplicação do ingrediente ativo fluazinam no controle de Sclerotinia sclerotiorum e sua influência na produtividade da cultura da soja de estudos conduzidos no Brasil entre 2010 e 2016.

Tabela 1. Estimativa metanalítica, $\mathrm{P}$ valor, limites inferiores e superiores, índice $\mathrm{I}^{2} \mathrm{e}^{2}$ de estudos utilizando o fungicida fluazinam ${ }^{\circledR}$ no controle de Sclerotinia sclerotiorum na cultura da soja.

\begin{tabular}{lllllll}
\hline Variável & Estimativa metanalítica & P valor & Limite inferior & Limite superior & $\boldsymbol{I}^{2}(\%)$ & H \\
\hline Produtividade $\left(\mathrm{Kg}^{2} \mathrm{ha}^{-1}\right)$ & 413.9 & $>.001^{*}$ & 344.6 & 483.1 & 85.1 & 6.73 \\
\hline
\end{tabular}

* Significativo ao nível de $1 \%$.

período de dez dias (26), a redução de incidência em plantas com aplicação de fluazinam pode favorecer os cultivos posteriores.

A estimativa metanalítica obtida no estudo indicou que houve acréscimo em produtividade com a utilização do fungicida fluazinam, contudo, os valores do índice $I^{2}(85,14 \%)$, indicaram valores altos de heterogeneidade, conforme sugerem Borenstein et al. (5). O valor obtido pelo índice $\mathrm{H}^{2}(6,73)$ indicou que o valor apresentado pela medida metanalítica sofreu influência da variação oriunda dos trabalhos. Os mesmos autores citam que para valores acima de 1,5 devem-se ser considerados os fatores causadores de efeito. Neste caso, foram desenvolvidas variáveis moderadoras de efeito.

\section{Moderadoras de efeito}

Do total de 50 entradas utilizadas na metanálise de modelos aleatórios, 24 entradas atendiam os critérios definidos para incidência em plantas e 21 entradas para severidade. De acordo com os diferentes níveis de incidência e severidade contidos no tratamento testemunha dos trabalhos inseridos na metanálise, foram formados dois grupos de estudos para cada covariável, experimentos que possuem incidência maior e menor que $35 \%$ e experimentos contendo severidade maior e menor que $15 \%$.

Os resultados metanalíticos demonstraram que não houve diferença significativa para a resposta de produtividade para as subclasses de incidência e severidade. Assim, o nível de contribuição da aplicação foliar de fluazinam na produtividade, se comparado à testemunha sem aplicação, não dependeu do nível e incidência da doença.

\section{Probabilidade de incremento em Produtividade}

A partir da estimativa metanalítica, valores de dispersão dos estudos e assumindo-se que o conjunto apresentava distribuição normal, efetuou-se a estimativa da probabilidade do ganho em produtividade com a utilização do fungicida fluazinam para o controle de mofo branco da soja (Figura 3).

Os resultados apresentaram 10,9\% de chances de não haver incremento em produtividade com a utilização de fluazinam para o controle de mofo branco da soja. Em contrapartida, estimou-se 89,1\% de chances de haver incremento de produtividade, independente da magnitude da mesma, quando utilizado o controle químico. Assim, constatou-se maior probabilidade de haver benefícios na produtividade com o manejo químico de mofo branco.

Notou-se ainda, conforme esperado, que quanto maior o incremento esperado na produtividade, menor foi a probabilidade de ocorrer este incremento. Ou seja, para incrementos da ordem de 120, 300, 600, 900 e $1.200 \mathrm{Kg} \cdot \mathrm{ha}^{-1}$ as probabilidades foram 82,8, 69,6, 41,7, 17,6 e 4,9\%, respectivamente. Paul et al. (18), estudando a influência de tratamentos com fungicidas na cultura do trigo, constataram que à medida em que se eleva a resposta em produtividade estimada, a probabilidade de ocorrência diminui até um ponto de equilíbrio onde não se há mais benefício

\section{Retorno financeiro}

O cálculo da probabilidade de retorno financeiro com a aplicação fungicida usou como base o preço de $\mathrm{R} \$ 50,00$ por saca de $60 \mathrm{~kg}$ de soja e o custo de $\mathrm{R} \$ 170,00 \mathrm{ha}^{-1}$ para duas aplicações do produto comercial, que contém o fluazinam como ingrediente ativo, equivalente a 3,4 sacas de soja $(204 \mathrm{Kg})$ de custo por hectare. A probabilidade de equilíbrio nos custos foi de 77,1\% (Figura 3).

Uma vez que o valor da soja e o custo do fungicida variam por condições de mercado, a estimativa não pode ser considerada um valor fixo, entretanto, serve de base para a compreensão do retorno proporcionado pela utilização de tratamento fungicida com fluazinam.

A aplicação de fluazinam aumentou a produtividade da soja atacada por S.scleotiorum, com estimativa metanalítica de $413,9 \mathrm{~kg} . \mathrm{ha}^{-1}$ $(\mathrm{p}<0.001)$ e amplitude variando de $344,6 \mathrm{~kg} \cdot \mathrm{ha}^{-1} \mathrm{a} 483,1 \mathrm{~kg} \cdot \mathrm{ha}^{-1}$, quando utilizado fungicida fluazinam no controle do mofo branco em soja na região centro-sul do estado do Paraná. Além disso, dado a estimativa de 77,1 \% de chances de equilíbrio econômico, a aplicação de fluazinam é uma técnica viável de manejo. 


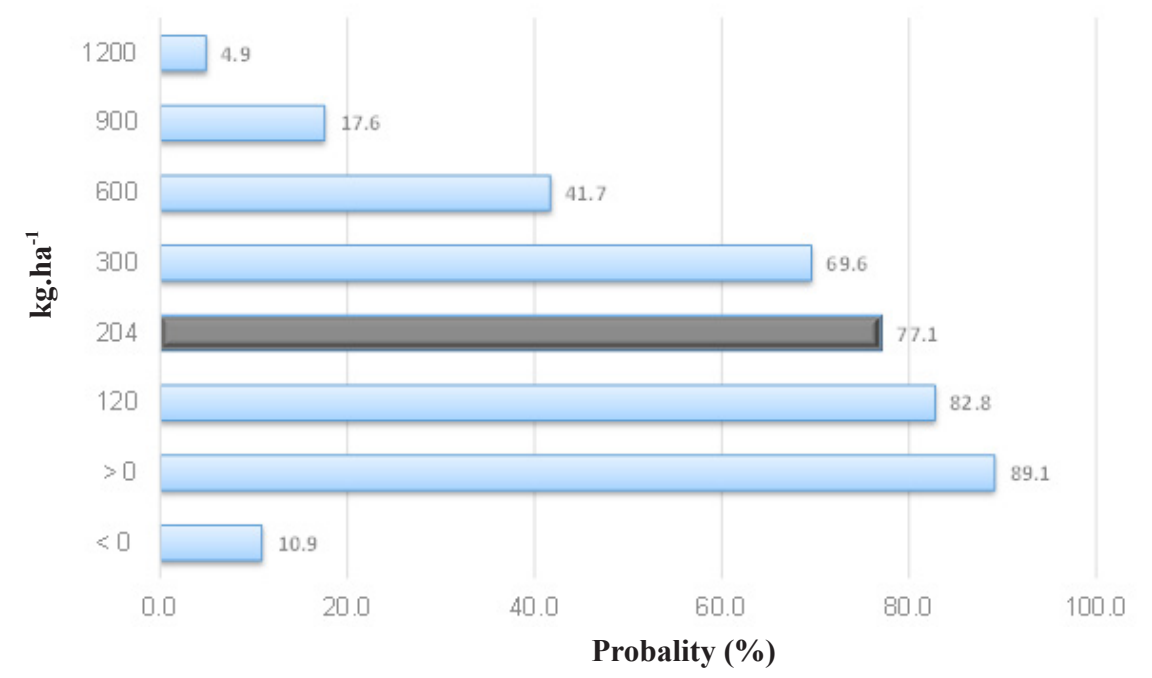

Figura 3. Probabilidade de acréscimo em produtividade com aplicação de fluazinam para o controle de Sclerotinia sclerotiorum na região sul do Brasil.

\section{REFERÊNCIAS}

1. Adams, D. C.; Gurevitch, J.; Rosenberg, M. S. Resampling tests for meta-analysis of ecological data. Ecology, London, v. 78, n. 5, p.1277-1283, 1997.

2.Almeida, A.M.R.; Ferreira, L.P.; Yorinori, J.T.; Silva, J.F.V.; Henning, A.A.; Godoy, C.V.; Costmilan, L.M.; Meyer, M.C. Doenças da soja. In: Kimati, H. Manual de fitopatologia. 4. ed. São Paulo: Agronômica Ceres, 2005. v.2. p.569-588

3. Bianchini, A.; Maringoni, A.C.; Carneiro, S.M.T.P.G. Doenças do feijoeiro. In: Kimati, H. Manual de fitopatologia. 4. ed. São Paulo: Agronômica Ceres. V.2 Doenças de Plantas cultivadas, 2005. p. 333-349.

4. Boland, G.J.; Hall, R. Index of plant hosts Sclerotinia sclerotiorum. Canadian Journal of Plant Pathology, London, v.6, p. 93-100, 1994.

5. Borenstein, M.; Hedges, L.V.; Higgins, J.P.T.; Rothstein, H.R. Introduction to meta-analysis. Chichester: Wiley and Sons, 2009.

6. Calvo, N.S.; Fantin, L.H.; Canteri, M.G.; Sumida, C.H. Modelo metanalítico na avaliação do incremento de produtividade com o uso de fungicidas para o controle de Sclerotinia sclerotiorum. ASB jornal, Londrina, v. 2, n. 1, p. 7 - 12, 2016.

7. COMPANHIA NACIONAL DE ABASTECIMENTO (CONAB). Acompanhamento da safra brasileira de grãos, v. 9 Safra 2015/16 - nono levantamento, Brasília, junho 2016. Disponível em: < http://www.conab. gov.br/OlalaCMS/uploads/arquivos/16_06_09_16_49_15_boletim_graos junho_2016_-_final.pdf $>$ Acesso em :13 de junho de 2016.

8. Fantin, L.H.; Canteri, M.G.; Silva, A.L. da.; Tupich, F.L.B.; Madden, L.V. Contribution of soybean seed treatment with Fluquinconazole to manage yield losses caused by Phakospora pachyrhizi using meta-analysis. African Journal of Agricultural Research. v.11, n.39, p.3880-3888, 2016.

9. Higgins, J.P.; Thompson, S.G. Quantifying heterogeneity in a meta-analysis. Statistics in Medicine. Chichester, v. 21, p.1539-1558, 2002.

10. Leite, R.M.V.B.C. Doenças do girassol. In: Kimati, H. Manual de fitopatologia. 4. ed. São Paulo: Agronômica Ceres, 2005, v. 2. p. 385-399.

11. Madden, L.V.; Paul, P.A. Meta-analysis for evidence synthesis in plant pathology: an overview. Phytopathology, Saint Paul, v.101, p. 16-30, 2011.

12. Massola, N.S.; Krugner, T.L. Fungos fitopatogênicos. In: Amorim, L.; Rezende, J.A.M.; Bergamim Filho, A. Manual de fitopatologia. 4. ed. São Paulo: Agronômica Ceres, 2011. v.1. p.149-206.

13. Meyer, M. C. Ensaio cooperativo de controle químico de mofo-branco em soja. Relatório Técnico Final. Londrina: EMBRAPA. 2012. 3 p.

14. Meyer, M.C.; Campos, H.D.; Godoy, C.V.; Utiamada, C.M. Ensaios cooperativos de controle químico de mofo branco na cultura da soja: safras 2009 a 2012. Londrina: Embrapa Soja, 2014. 101p. Doc. 345.

15. Meyer, M.C.; Campos, H.D.; Godoy, C.V.; Utiamada, C.M. Ensaios cooperativos de controle biológico de mofo-branco na cultura da soja: safras
2012 a 2015. 100 p Londrina: Embrapa Soja. 2016. Doc. 368.

16. Ngugi, H. H.; Esker, P. D.; Scherm, H. Meta-analysis to determine the effects of plant Disease Management Measures: Review and Case Studies on Soybean and Apple. Phytopathology, Saint Paul, v.101, n. 1, p.31-41, 2011.

17. Paul, P.A.; Lipps, P.E.; Hershman, D.E.; McMullen, M.P.; Draper, M.A.; Madden, L.V. Efficacy of triazole-based fungicides for fusarium head blight and deoxynivalenol control in wheat: A multivariate meta-analysis. Phytopathology, Saint Paul, v.98, p, 999-1011. 2008.

18. Paul, P.A.; Mcmullen, M.P.; Hershman, D.E.; Madden, L.V. Meta-analysis of the effects of Triazole-Based Fungicides on Wheat Yield and test Weight as Influenced by Fusarium Head Blight Intensity. Phytopathology, Saint Paul, v. 100, p.160-171, 2010.

19. Paul, P. A.; Madden, L. V.; Bradley, C. A.; Robertson, A. E.; Munkvold, G. P.; Shaner, G.; Esker, P. Meta-analysis of yield response of hybrid field corn to foliar fungicides in the U.S. Corn Belt. Phytopathology, Saint Paul, v. 101, p.1122-1132, 2011.

20. R Development Core Team : A language and environment for statistical computing. R Foundation for Statistical Computing, Vienna. Disponível em: <http://www.R-project.org/>. 2011.

21. Rodrigues, C.L.; Ziegelman, P.K. Metanálise: um guia prático. Revista HCPA, Porto Alegre, v.30, n. 4, p. 436-447, 2010.

22. Salam, K. P.; Thomas, G. J.; Beard. C.; Loughman, R.; MacLeod, W.J.; Salam, M.U. Application of meta-analysis in plant pathology: a case study examining the impact of fungicides on wheat yield loss from the yellow spot-septoria nodorum blotch disease complex in Western Australia. Food Security, London, v.5, p. 319-325, 2013.

23. SEAB/DERAL. Secretaria de Estado da Agricultura e do Abastecimento, Departamento de Economia Rural. Disponível em: < http://www.agricultura.pr.gov.br/arquivos/File/deral/Prognosticos/2016/Soja_2015_16.pdf $>$. Acesso em: 10 maio 2016

24. Scherm, H.; Thomas, C.S.; Garrett, G.; Olsen, J.M. Meta-analysis and other approaches for synthesizing structured and unstructured data in plant pathology. Annual Review of Phytopathology, Saint Paul, v. 52, p. 453-476. 2014.

25. Schwartz, H.F.; Steadman, J.R. White mold. In: Schwartz, H.F.; Pastor-Corrales, M. A. (Ed.). Bean production problems in the tropics Cali. Cali: Centro Internacional de Agricultura Tropical, 1989. p. 211-230.

26. Steadman, J. R. White mold: a serious yield-limiting disease of bean. Plant Disease, Saint Paul, v.67, n.4, p. 346-350, 1983.

27- Tupich, F.L.B. Meta-análise do efeito de Fluazinam na produtividade influenciada pela intensidade de Sclerotinia sclerotiorum (Lib de Bary). 2015. 64f. Dissertação (Mestrado em Agronomia) 
- Universidade Estadual de Londrina, Londrina.

28. Viechtbauer, W. Conducting meta-analyses in $\mathrm{R}$ with the metafor package. Journal of Statistical Software, Los Angeles, v.36, n.3, p. 1-48, 2010. Disponível em: <http://www.jstatsoft.org/v36/i03/>. Acesso em: 13 de junho de 2016
29. Wutzki, R.C.; Jaccoud Filho, D.S.; Berger Neto, A.; Tullio, H.E.; Juliatti, F.C.; Nascimento, A.J. Reduction of white mold level on soybean by fungicide management strategies. Bioscience Journal, Uberlândia, v.32, n.3, p.642-651, 2016. 